\title{
Dynamics of Allelopathic Two Species Model Having Delay in Predation
}

\author{
${ }^{1}$ M.A.S.Srinivas, ${ }^{2}$ B S N Murthy , ${ }^{1}$ A.Prasanthi \\ 'Professor., Department of Mathematics, JNTUH,TS,India-500085. \\ ${ }^{2}$ Assoc.prof., Department of Mathematics, SSAIS\&T, A.P, India-533001.
}

\begin{abstract}
In this paper, we have considered an allelopathic model of two species and discussed the dynamics of the model when the effect of interaction on prey is based on the rate of consumption of the prey by the predator and the rate of release of toxicant by the predator. The interaction of the predator and prey results on the growth of predator after a time interval $(\tau)$. It is shown that the time delay can cause a switch from stable state to unstable state and there by Hopf-bifurcation occurs.
\end{abstract}

Key words: Hopf-bifurcation, Prey-Predator, Stability, Time-delay, Toxicant.

\section{Introduction}

Lotka [1] and Volterra [2] initiated the research in the field of theoretical ecology. Since then many researchers studied the Predator-Prey or competitive model with Mutualism and commensalism. The dynamical relationship between predators \& their prey is one of the important aspect in the population dynamics. There has been great interest in dynamical characteristics like stable, unstable \& oscillatory behavior. The problem of harvesting two ecological independent \& logistically growing fish species was studied by C.W. clark [3], Brauer and Soudack [4, 5], Dai \& Tang [6] , Myerscough [7].

Allelopathy can be defined as the direct or indirect harmful effect of one species on another through the production of a chemical released into the environment. In recent times many researchers are extensively studying the eco-toxicological effects of toxicants released by the marine biological species among themselves. An example of this kind is the toxicant produced by the Uni celluar green alga chlorella vulgaris. The toxicant limits the size of it's own population and also inhabits growth of the Planktonic algaes, Asterionella Formosa and Nitzschia frustulum.Maynardsmith[8],Chattopadhyay [9] studied a two species Lotka-Volterra competitive system, in which toxic substance produced by one species effects the other.They also studied the stability properties of the system.

Das $[10,11]$ studied \& analyzed the harvesting of fish species, as aPrey -Predator model in the presence of a toxin released by some other source. In their work a catch-rate function is defined in place of the usually catch-per unit effort hypothesis for the problem of non-selective harvesting. Kar and Chaudhuri(9) proposed a model for two competing fish species in the presence of toxin and Combined harvesting of the species, keeping in view the Maynard smith conjecture. As Maynard smith's conjecture is valid for the large classes of marine species, the model proposed by Kar and Chaudhuri (9) is applicable to large class of other marine species. R.P.Gupta etal . [13] extended the models proposed by Kar and Chaudari [12] for any two populations having competition and harvested by different agencies with different harvesting efforts. They discussed the existence of two saddle-node bifurcations using Sotomayor's theorem.

In nature there are large classes of marine species other than fish such as Algae and bacterial that produce toxic substance which effect the other species while competing for food [14]. But in some cases the toxic substance released by one species may not effect the other species immediately, but with some delay in time.

In this paper we propose a two species Prey-Predator model in which the two species having densities $z_{1}(t), z_{2}(t)$ are harvested by different agencies with harvesting efforts $H_{1} \& H_{2}$ respectively and the corresponding catchability coefficients of the two species being $C_{1}, C_{2}$. In this proposed dynamical model the two species obey law of logistic growth with intrinsic growth rates $r, s$ and have carrying capacities K, L .Both the species release toxic substances which affect the other, the toxic coefficients of prey \& predator are respectively $\eta_{1}, \eta_{2}$. The predator population has food source other than the prey. The effect of interaction of the species on prey is based on the rate of consumption of the prey by the predator and the rate of release of toxicant by the predator. The net rate of effect of interaction on prey is denoted by $a_{1}$. Similarly the effect of interaction 
of the species on predator is based on the rate of its predation and the rate of release of toxicant by the prey. The net rate of effect of interaction on predator is denoted by $a_{2}$. All these parameters are assumed to be positive.

It is well known that in some prey-predator systems the rate of change in the predator depends on numbers of prey and predator at time $t$ and in some other systems the rate of change in the predator depends on prey \& predators population present at some previous times say $(\mathrm{t}-\tau)$.In this paper we studied the changes occurred in stability of the dynamical system when a delay $(\tau)$ is incorporated in predation term. The theoretical results are validated by the numerical simulations.

\section{Mathematical Model}

The mathematical formulation of the toxicant prey-predator dynamical problem with different harvesting efforts is

$$
\left.\begin{array}{l}
\frac{d z_{1}}{d t}=r z_{1}\left(1-\frac{z_{1}}{K}\right)-a_{1} z_{1} z_{2}-\eta_{1} z_{1}^{2} z_{2}-C_{1} H_{1} z_{1} \\
\frac{d z_{2}}{d t}=s z_{2}\left(1-\frac{z_{2}}{L}\right)+a_{2} z_{1}(t) z_{2}(t)-\eta_{2} z_{1} z_{2}^{2}-C_{2} H_{2} z_{2}
\end{array}\right\}
$$

It is well known that in some prey-predator systems the rate of change in the predator depends on numbers of prey and predator at time $t$ and in some other systems the rate of change in the predator depends on prey \& predators population present at some previous times say $(\mathrm{t}-\tau$ ). By incorporating time delay $\tau$ in predation term, the equation (2.1.1) becomes

$$
\left.\begin{array}{l}
\frac{d z_{1}}{d t}=r z_{1}\left(1-\frac{z_{1}}{K}\right)-a_{1} z_{1} z_{2}-\eta_{1} z_{1}^{2} z_{2}-C_{1} H_{1} z_{1} \\
\frac{d z_{2}}{d t}=s z_{2}\left(1-\frac{z_{2}}{L}\right)+a_{2} z_{1}(t-\tau) z_{2}(t-\tau)-\eta_{2} z_{1} z_{2}^{2}-C_{2} H_{2} z_{2}
\end{array}\right\}
$$

From biological point of view we only interested on the interior equilibrium $\mathrm{E}\left(z_{1}^{*}, z_{2}^{*}\right)$.

$$
\text { Let } Z_{1}=z_{1}-z_{1}^{*}, Z_{2}=z_{2}-z_{2}^{*} \text { be the perturbed variables }
$$

After removing the non-linear terms we obtain linearaized system corresponding to (2.1.2) is

$$
\left.\begin{array}{l}
\frac{d Z_{1}}{d t}=\left[r-\frac{2 r}{K} z_{1}^{*}-a_{1} z_{2}^{*}-2 \eta_{1} z_{1} z_{2}^{*}-c_{1} H_{1}\right] Z_{1}+\left[-a_{1} z_{1}^{*}-\eta_{1} z_{1}^{* 2}\right] Z_{2} \\
\left.\frac{d Z_{2}}{d t}=\left[-\eta_{2} z_{2}^{* 2}+a_{2} e^{-\mu \tau} z_{2}^{*}\right] Z_{1}+\left[s-\frac{2 s}{L} z_{2}^{*}-2 \eta_{2} z_{1}^{*} z_{2}^{*}-c_{2} H_{2}+a_{2} e^{-\mu \tau} z_{1}^{*}\right] Z_{2}\right]
\end{array}\right\}
$$

The characteristic equation of the linear system is given by

$$
\begin{aligned}
& \Delta(\mu, \tau)=\mathrm{X}(\text { 回 })+\mathrm{Y}(\text { 回 }) e^{-\mu \tau}=0 \\
& \begin{array}{ll}
\text { Where } \mathrm{X}(\mu)=\mu^{2}+\mathrm{P} \mu+\mathrm{R}, \mathrm{Y}(\mu)=\mathrm{Q} \mu+\mathrm{S} \\
\mathrm{P}=-\mathrm{A}-\mathrm{D} & \mathrm{A}=r-\frac{2 r}{K} z_{1}^{*}-a_{1} z_{2}^{*}-2 \eta_{1} z_{1}^{*} z_{2}^{*}-c_{1} H_{1} \\
\mathrm{Q}=-a_{2} z_{1}^{*} & \mathrm{~B}=-a_{1} z_{1}^{*}-\eta_{1} z_{1}^{* 2} \\
\mathrm{R}=\mathrm{AD}-\mathrm{BC} & \mathrm{C}=-\eta_{2} z_{2}^{* 2} \\
\mathrm{~S}=\left(A z_{1}^{*}-B z_{2}^{*}\right) a_{2} & \mathrm{D}=s-\frac{2 s}{L} z_{2}^{*}-2 \eta_{2} z_{1}^{*} z_{2}^{*}-c_{2} H_{2}
\end{array}
\end{aligned}
$$


We discuss the stability of the system at the interior equilibrium $\mathrm{E}\left(z_{1}^{*}, z_{2}^{*}\right)$.

Case 1: when $\tau=0$

\section{Stability Analysis}

In the absence of discrete time delay we investigate the stability of the system (2.1.2) around the interior equilibrium (E) . The system (2.1.2) becomes a system of ordinary differential Equations (2.1.1),then the corresponding characteristic equation is

$X(\mu)+Y(\mu)=0$.

$$
\begin{gathered}
\text { i. } e,\left(\mu^{2}+P \mu+R\right)+(Q \mu+S)=0 \\
\mu^{2}+(P+Q) \mu+(R+S)=0
\end{gathered}
$$

Sum of the roots $=-(\mathrm{P}+\mathrm{Q})<0$

Product of the roots $=\mathrm{R}+\mathrm{S}$

$\mathrm{R}+\mathrm{S}>0$ if $\frac{a_{1} L}{s}<\frac{\eta_{1}}{\eta_{2}}<\frac{r}{K a_{2}}$

We can say that both the roots of (3.1.1) are real \& negative (or) complex conjugate with negative real part iff $\mathrm{P}+\mathrm{Q}>0 \& \mathrm{R}+\mathrm{S}>0$.

(3.1.2)

Hence, in the absence of time delay, the system is locally asymptotically stable when $\frac{a_{1} L}{s}<\frac{\eta_{1}}{\eta_{2}}<\frac{r}{K a_{2}}$ is valid.

Theorem 3.1: In the absence of the delay, the system (2.1.1) is locally asymptotically stable at the $\left.E\left(z_{1}^{*}, z_{2}^{*}\right)\right)$ iff $\frac{a_{1} L}{s}<\frac{\eta_{1}}{\eta_{2}}<\frac{r}{K a_{2}}$

We augment our analytical findings through numerical simulations by the following example.

\section{Example 1}

The parameters in the model (2.1.1) are taken as

$\mathrm{r}=4, \mathrm{~s}=5, a_{1}=0.1, a_{2}=0.9, \eta_{1}=0.008, \eta_{2}=0.005, c_{1}=0.1, c_{2}=0.2, H_{1}=30, H_{2}=40, \mathrm{~K}=500, \mathrm{~L}=400$.

Initial values of the species are $z_{1}=10, z_{2}=5$ and $\tau=0$

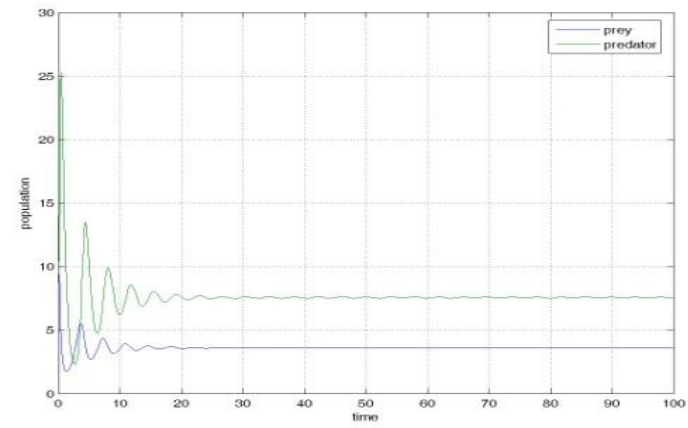

Fig.1 show the variation of populations against time.

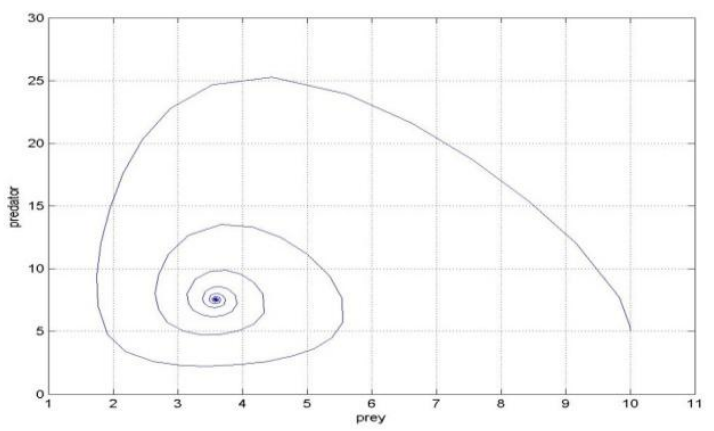

Fig.2 show the phase- portraits of prey and predator 
Case 2: when $\tau>0$,

Let $\mu(\tau)=\alpha(\tau)+i \theta(\tau)$ be a root of the characteristic equation (2.1.4).

Let $\tau$ be a particular value of the delay such that $\alpha(\tau)=0, \theta(\tau)>0$

Put $\mu=i \theta$ in (2.1.4) we get

$\left[(\mathrm{i} \theta)^{2}+\mathrm{P}(\mathrm{i} \theta)+\mathrm{R}\right]+[\mathrm{Q}(\mathrm{i} \theta)+\mathrm{s}] e^{-i \theta \tau}=0$

$\mathrm{i}^{2} \theta^{2}+\mathrm{P}(\mathrm{i} \theta)+\mathrm{R}+[\mathrm{Q}(\mathrm{i} \theta)+\mathrm{S}]\{\cos \theta \tau-\mathrm{i} \sin \theta \tau\}=0$

$-\theta^{2}+\mathrm{p}(\mathrm{i} \theta)+\mathrm{R}+[\mathrm{iQ} \theta+\mathrm{S}]\{\cos \theta \tau-\mathrm{i} \sin \theta \tau\}=0$

Separating the real \& imaginary parts, we get

$\theta^{2}-\mathrm{R}=\mathrm{S} \cos \theta \tau+\mathrm{Q} \theta \sin \theta \tau$

$\mathrm{P} \theta=\mathrm{S} \sin \theta \tau-\mathrm{Q} \theta \cos \theta \tau$

Squaring \& adding these two equations, we get the fourth order equation

$\theta^{4}+\theta^{2}\left(\mathrm{P}^{2}-2 \mathrm{R}-\mathrm{Q}^{2}\right)+\left(\mathrm{R}^{2}-\mathrm{S}^{2}\right)=0$

Sub case 1: If $P^{2}-2 R-Q^{2}>0$ and $R^{2}-S^{2}>0$ then the equation (3.1.4) does not have any real solutions.

Hence this case is omitted as $\theta(\tau)$ is a real number.

Sub case 2: If $P^{2}-2 R-Q^{2}>0$ and $R^{2}-S^{2}<0$ then the equation (3.1.4) have a unique positive root, it is $\theta_{0}^{2}$ and let the corresponding $\tau$ be $\tau_{0}$.

Sub case 3: If $P^{2}-2 R-Q^{2}<0, R^{2}-S^{2}>0$ and $\left(P^{2}-2 R-Q^{2}\right)^{2}-4\left(R^{2}-S^{2}\right)>0$ then the equation (3.1.4) have two positive roots. Let them be $\theta_{ \pm}^{2}$ and the corresponding $\tau$ be $\tau_{n}^{ \pm}$.

The above said positive roots, either from sub case 2 or from sub case 3 ,satisfy all the equations from (3.1.3) to (3.1.4).

Eliminating $\sin \theta \tau$ from (3.1.3), we get

$\tau_{k}=\frac{1}{\theta} \cos ^{-1}\left[\frac{\theta^{2}(\mathrm{~S}-P Q)-R S}{S^{2}+Q^{2} \theta^{2}}\right]+\frac{2 k \pi}{\theta}$ wherek $=0,1,2 \ldots$

Now differentiating equation (2.1.4) w.r.t $\tau$, we obtain

$\left[2 \mu+P+Q e^{-\mu \tau}-\tau(Q \mu+S) e^{-\mu \tau}\right] \frac{d \mu}{d \tau}=\mu e^{-\mu \tau}(Q \mu+S)$

$\left[\frac{d \mu}{d \tau}\right]^{-1}=\frac{2 \mu+P}{-\mu\left(\mu^{2}+P \mu+R\right)}+\frac{Q}{\mu(Q \mu+S)}-\frac{\tau}{\mu}$

$\operatorname{Re}\left[\frac{d \mu}{d \tau}\right]^{-1}=\frac{2\left(\theta^{2}-R\right)+P^{2}}{\theta^{4}+\left(P^{2}-2 R\right) \theta^{2}+R^{2}}-\frac{Q^{2}}{S^{2}+Q^{2} \theta^{2}}$

Thus,

$$
\begin{aligned}
\operatorname{Sign}\left[\frac{d}{d \tau}(\operatorname{Re} \mu)\right] & =\operatorname{Sign}\left[\operatorname{Re}\left(\frac{d \mu}{d \tau}\right)^{-1}\right]_{\mu=i \theta} \\
= & \operatorname{Sign}\left[\frac{2 \theta^{2}+\left(P^{2}-2 R-Q^{2}\right)}{Q^{2} \theta^{2}+S^{2}}\right]
\end{aligned}
$$

Note that the $\theta$ may be $\theta_{0}$ or $\theta_{ \pm}$

Theorem 3.2: In the presence of the delay, the system (2.1.2) is locally asymptotically stable at $E\left(z_{1}^{*}, z_{2}^{*}\right)$ iff $R^{2}-S^{2}<0$ for all $\tau<\tau_{0}$.It is unstable for all $\tau>\tau_{0}$ and hopf - bifurcation occurs at $\tau=\tau_{0}$

Proof:

From equation (3.1.8) we have 


$$
\begin{aligned}
& \operatorname{Sign}\left[\frac{d}{d \tau}(\operatorname{Re} \mu)\right]=\operatorname{Sign}\left[\operatorname{Re}\left(\frac{d \mu}{d \tau}\right)^{-1}\right]_{\mu=i \theta_{0}}=\operatorname{sign}\left[\frac{2 \theta_{0}^{2}+\left(P^{2}-2 R-S^{2}\right)}{Q^{2} \theta_{0}^{2}+S^{2}}\right] \\
& \operatorname{sign}\left[\operatorname{Re}\left(\frac{d \mu}{d \tau}\right)\right]_{\mu=i \theta_{0}}=\operatorname{sign}\left[\operatorname{Re}\left(\frac{d \mu}{d \tau}\right)^{-1}\right]_{\theta=\theta_{0}, \tau=\tau_{0}}>0
\end{aligned}
$$

This signify that there exits Eigen values with negative real part for $\tau<\tau_{0}$ and there exits Eigen values with positive real part for $\tau>\tau_{0}$. Therefore, the transversality condition holds and hence hopf-bifurcation occurs at $\theta=\theta_{0}, \tau=\tau_{0}$

Theorem 3.3: In the presence of delay, the system is locally asymptotically stable at $E\left(z_{1}^{*}, z_{2}^{*}\right)$ iff $P^{2}-2 R-Q^{2}<0, R^{2}-S^{2}>0$ and $\left(P^{2}-2 R-Q^{2}\right)^{2}-4\left(R^{2}-S^{2}\right)>0$ forall $\tau \varepsilon\left[0, \tau_{0}^{+}\right) \bigcup\left(\tau_{0}^{-}, \tau_{1}^{+}\right) \bigcup \ldots \bigcup\left(\tau_{m-1}^{-}, \tau_{m}^{+}\right)$.

It is unstable forall $\tau \varepsilon\left[\tau_{0}^{+}, \tau_{0}^{-}\right) \bigcup\left(\tau_{1}^{+}, \tau_{1}^{-}\right) \bigcup \ldots \bigcup\left(\tau_{m-1}^{+}, \tau_{m}^{-}\right)$for some positive integer $\mathrm{m}$.

\section{Proof:}

From (3.1.8) it follows that

$$
\operatorname{sign}\left\{\left[\frac{d}{d \tau}(\operatorname{Re} \mu)\right]_{\mu=i \theta_{+}}\right\}=\operatorname{sign}\left\{+\frac{\sqrt{\left(\mathrm{P}^{2}-2 R-Q^{2}\right)^{2}-4\left(R^{2}-S^{2}\right)}}{\left\{\left(-\theta_{+}^{2}+R\right)^{2}+P^{2} \theta_{+}^{2}\right\}\left\{S^{2}+Q^{2} \theta_{+}^{2}\right\}}\right\}
$$

Therefore, $\left\{\frac{d}{d \tau}(\operatorname{Re} \mu)\right\}_{\theta=\theta_{+}, \tau=\tau_{n}^{+}}>0$.

Again, $\operatorname{sign}\left\{\left[\frac{d}{d \tau}(\operatorname{Re} \mu)\right]_{\mu=i \theta_{-}}\right\}=\operatorname{sign}\left\{-\frac{\sqrt{\left(\mathrm{P}^{2}-2 R-Q^{2}\right)^{2}-4\left(R^{2}-S^{2}\right)}}{\left\{\left(-\theta_{-}^{2}+R\right)^{2}+P^{2} \theta_{-}^{2}\right\}\left\{S^{2}+Q^{2} \theta_{+}^{2}\right\}}\right\}$

Therefore, $\left\{\frac{d}{d \tau}(\operatorname{Re} \mu)\right\}_{\theta=\theta_{-}, \tau=\tau_{n}^{-}}<0$.

Hence we have $\left[\frac{d}{d \tau}(\operatorname{Re} \mu)\right]_{\tau=\tau_{n}^{+}}>0$ and $\left[\frac{d}{d \tau}(\operatorname{Re} \mu)\right]_{\tau=\tau_{n}^{-}}<0$ Hence, the transversality conditions are satisfied . This completes the proof.

We augment our analytical findings through numerical simulations by the following examples.

\section{Example 2}

The parameters of the model (2.1.2) are taken as $\mathrm{r}=4, \mathrm{~s}=5, a_{1}=0.1, a_{2}=0.9, \eta_{1}=0.008, \eta_{2}=0.005, c_{1}=0.1, c_{2}=0.2, H_{1}=30, H_{2}=40, \mathrm{~K}=500, \mathrm{~L}=400$.

Initial values of the species are $z_{1}=10, z_{2}=5$ and $\tau=0.25<\tau_{0}=0.29$ 


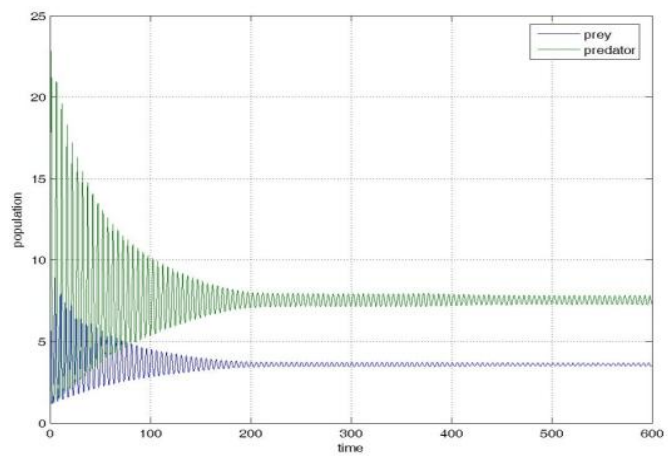

Fig.3 show the stable variation of populations against time

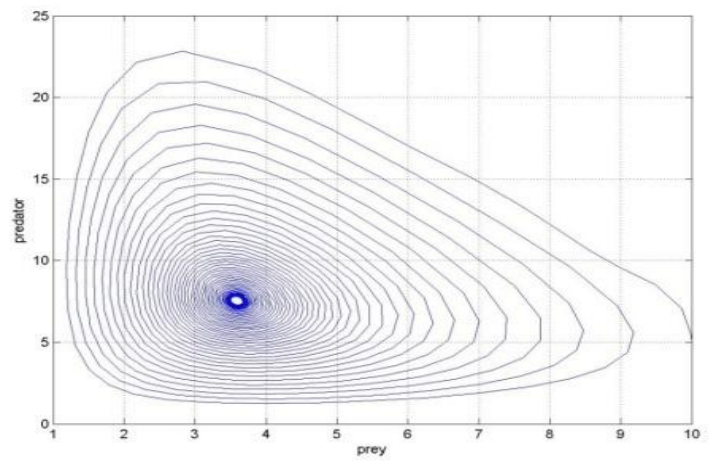

Fig.4 show the phase- portraits of prey and predator

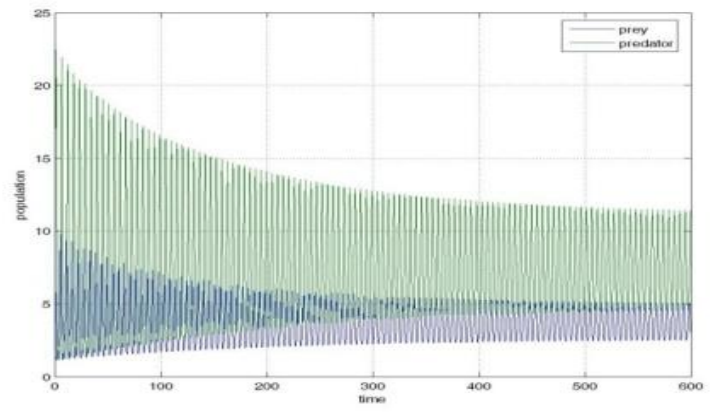

Fig.5 show the unstable variation of populations against time when $\tau=\tau_{0}=0.29$

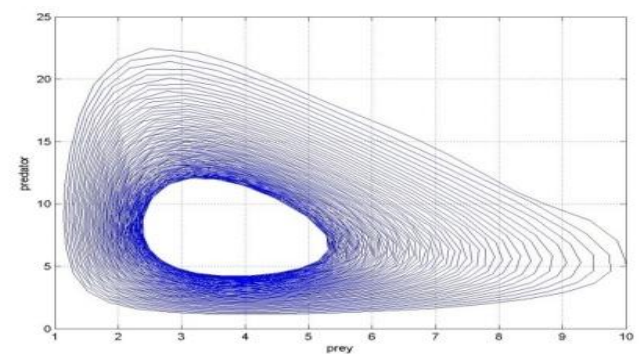

Fig.6 6 show the phase- portraits of prey and predator $\tau=\tau_{0}=0.29$ 


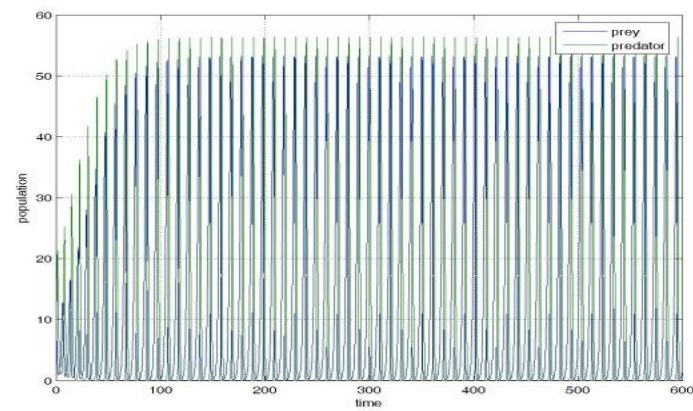

Fig.7 show the unstable variation of populations against time When $\tau=0.5>0.29$

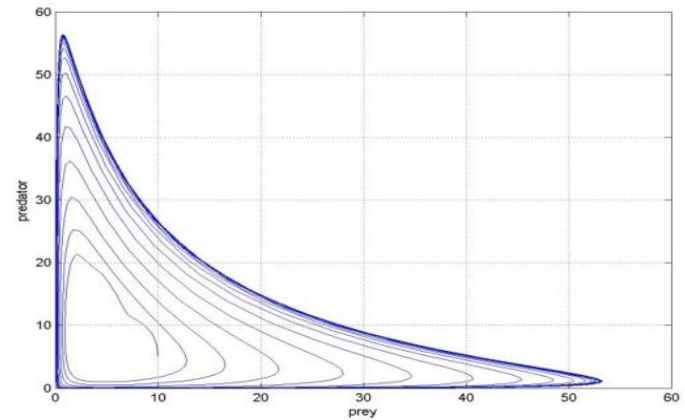

Fig.8: show the Phase portraits of prey and predator when $\tau=0.5>0.29$

\section{Concluding Remarks}

The model discussed here has the following characteristics

(a) The prey population decreases not only by predation but also by the toxic substance released by the predator. (b) The predator population also decreases by the toxic substance released by the prey population. Hence the model has not only the prey-predator relationship but also the two species have effects of toxicants released by them on each other.

It is observed that delay of all dimensions does not induce any instability; the delay of certain dimensions can induce instability oscillation via Hopf bifurcation. It is also observed that switching of stability occurs. The effect of delay incorporated in the predation term is clearly studied, and the theoretical results obtained are validated through the numerical simulations using MATLAB.

\section{Acknowledgements:}

This research is funded by University grants commission, India,(Grant no 42-18/2013(SR)).

\section{References}

[1]. A. J. Lotka, Elements of Physical biology, Williams and Wilkins, Baltimore, 1925.

[2]. V.Volterra, Leconssen la theorie mathematique de la leitte pou lavie, Gauthier- Villars, Paris, 1931

[3]. Clark C.W, mathematical Bio economics: The optimal management of Renewable Resources, wiley, New York , 1976.

[4]. Brauer .F and A.C. Soudack, stability regions and transition phenomena for harvested predator - prey systems. J.math. Biol.7,(319337)(1979).

[5]. Brauer .F and A.C. Soudack, stability regions and transition phenomena for harvested predator - prey systems. J.math. Biol.7,(319337)(1979).

[6]. Dai.G, Tang.M, coexistence region and global dynamics of a harvested predator - prey systems. SIAM J Appl.math 58:193-210,( 1998).

[7]. Myerscough.M.R,Gray.B.E, Hograth.W.L and Norbury.J, An analysis of an ordinary differential equation model for a two - species predator - prey system with harvesting and stocking , I.Math.Bzol.30.389-411(1992).

[8]. Maynard smith. J, models in Ecology, cambridge university press, London, 1974, P.146.

[9]. Chattopadhyay. J , Effect of toxic substances on a two species competitive system, Ecol. model. 84 pp.287-289 (1996).

[10]. Das.T, R.N. Mukherjee, and K.S. Chaudhuri, Harvesting of a prey - predator fishery in the presence of toxicity, Appl.math;33: 2282-2292,(2009).

[11]. Das.T, R.N. Mukherjee, and K.S. Chaudhuri, Bio economic harvesting of a prey - predator fishery , J. Biol.Dyn.3[5], pp 447462(2009)

[12]. Kar.T.K and chaudhuri. K.S,on non-selective harvesting of two competing fish species in the presence of toxicity Ecol.model. 161 pp. 125-137 (2003).

[13]. R.P.gupta, Malay Banerjee \& Peeyush Chandra , The dynamics of two - species allelopathic competition with optimal harvesting , Journal of Biological Dynamics , 6:2, 674-694.

[14]. P.Fergola , M. Cerasuolo, A.Pollio,G.Pinto,and M.Dellagreca, Allelopathy and competition between Chlorella Vulgaris and Pseudokirchneriella subcapitata: Experiments and mathematical model,Ecol.Model.208 (2007),pp.205-214 